\title{
Sur la piste des idées
}

L'ordre naturel de la vie est ainsi fait qu'il contraint les philosophes à consulter parfois un médecin, notamment dans leur grand âge, à un moment où leur sagesse a atteint un degré de plénitude élevé mais que des maux petits et grands se font jour. Un phénomène qui n'étonnera personne puisqu'après tout, les philosophes ne sont, eux aussi, que des êtres humains.

En revanche, la démarche consistant, pour un médecin, à se rendre chez un philosophe n'est pas habituelle, bien que la relation étroite entre les deux domaines apparaisse clairement et qu'elle fasse traditionnellement partie de la profession médicale depuis la nuit des temps. D’Hippocrate à Karl Jaspers ou Thure von Uexküll en passant par Galien et Paracelse, nombreux sont les médecins remarquables qui ont également été des philosophes ou ont suivi des cours de philosophie.

Réfléchir sur le monde (sur les hommes dans le monde et sur la pensée elle-même) est le propre de l'être humain. A l'instar des médecins praticiens, celles et ceux qui s'occupent de la personne dans sa globalité devraient disposer d'une certaine affinité naturelle pour les questions philosophiques ou la développer presque obligatoirement au cours de leur activité professionnelle.

L'inclusion de la philosophie dans le cursus professionnel de la médecine, tel qu'il a été conçu pendant des siècles, ne va plus de soi aujourd'hui, du moins à première vue. L'augmentation des connaissances liée aux progrès réalisés par une médecine axée sur les sciences a provoqué un degré de spécialisation pouvant empêcher de percevoir le tout. En tous les cas, il est devenu pratiquement impossible de fournir des «prestations de pointe» dans plusieurs domaines et de s'y faire un nom, p.ex. comme médecin et comme philosophe. Dans le quotidien de la profession médicale, il est encore plus difficile de faire l'équilibre entre la pression induite par les prestations, la bureaucratie, les mécanismes de contrôle et les contraintes économiques. La camisole de force que représente l'efficacité imposée ne nous laisse que peu d'espace libre pour mener des réflexions philosophiques, que ce soit avec les patients, les collègues ou pour soi-même.

Et pourtant: le besoin de l'être humain - et des médecins - de s'adonner à la philosophie reste vivant. Les articles publiés dans le BMS l'attestent régulièrement: ils sont souvent rédigés - notamment ces derniers temps - par des médecins qui ont également accompli des études de philosophie. A l'ère de la biologie moléculaire, il demeure fascinant de se mettre sur la piste des idées, certainement aussi parce que l'essence d'une idée ne se laisse pas saisir avec les méthodes de cette science.

Ce qui m'amène tout naturellement à mentionner l'article qui a suscité mes réflexions. A partir de la page 171 de la présente édition, vous trouverez une présentation du cursus «Philosophie pour professionnels de la médecine et de la psychothérapie», offert depuis trois ans par l'Université de Zurich. Les impressions et regards qu'il contient vous donneront peut-être envie d'en savoir plus. Kafka disait: «C'est en marchant que l'on crée des chemins.»

Bruno Kesseli

Philosophia. Détail de «Septem Artes Liberales» dans «Hortus deliciarium» de Herrade de Landsberg (env. 1180). 